\title{
The Disparity Information and Communication Technology for Developing Countries has in the Delivery of Healthcare Information
}

\author{
Prajesh N. Chhanabhai" and Alec Holt
}

Health Informatics, Department of Information Science, University of Otago, New Zealand

\begin{abstract}
Information and Communication Technologies (ICT) have merged into the world of healthcare slowly but surely. However, the marriage between the use of technology and its full impact in the health sector has not been fully realised. The focus of this paper is to highlight the impact of ICT on revolutionising access to healthcare information and thus quality of health for populations of the developing world. This paper highlights on the importance of being able to access health information and how traditional media methods have been utilised to allow this within a developing country setting, highlighting the clear digital divide. The paper then addresses the impact of convergent communication technologies and mobile technologies in providing a means of addressing existing healthcare problems within a developing country setting.
\end{abstract}

Keywords: Digital divide, developing countries, convergent communication.

\section{INTRODUCTION}

Information and communication technology (ICT) can be defined as tools that facilitate communication and the processing and transformation of data by electronic means [1]. This includes contemporary technologies such as the iPhone, Internet and social media tools to the more traditional radio and TV media.

The power of ICT is that it has the ability to change the way healthcare is conducted, this has a great impact as traditionally healthcare is a very regimented profession. However in a developing country setting the critical mass of professional and community users of ICTs in health has not yet been reached in all sectors, let alone the health sector [2]. It is in the developing world that ICTs can make and should make the highest impact. This is the population that probably requires most of the benefits that are provided by the use of ICTs. Benefits include [3]:

- Improved dissemination of public health information and facilitated public discourse around subjects that are major public health threats

- $\quad$ Enabled collaboration and cooperation among health workers

- Supported more effective health research and the dissemination and access to research findings

- Improved the efficiency of health administration

- Improve the ability to monitor outbreaks and have effective management plans

However with the inherent complexities of both technology and the developing country setting there can be no single solution that will work in all cases. The

*Address correspondence to this author at the Health Informatics, Department of Information Science, University of Otago, New Zealand; Tel: +64-3-4798316; Fax: +64-3-479-8311;

E-mail:pchhanabhai@infoscience.otago.ac.nz introduction of ICTs into this setting requires a transitional process that allows the people on the ground to feel the direct benefits. It cannot be seen as a political initiative that provides benefits to people in power only. According to Chetley there are seven conclusions that can be made for introducing ICT into the health sector that should be applicable to all levels irrespective of setting and complexity [3].

1. Keep the technology simple relevant and local

2. Build on what is already there

3. Involve users in the design

4. Strengthen capacity to use, work with and develop effective ICTs

5. Introduce greater monitoring and evaluation

6. Introduce communication strategies in the design of ICT projects

7. Continue to research and share learning about what works and what fails

Despite these conclusions, it must be remembered that the nature of healthcare systems is different to other sectors. In banking for example there are limited terms, limited transaction possibilities and very simple and standard information is needed about customers and most processes follow on some agreed national or international standard. The options within healthcare are much more complex due to the array of data types. A simple medical record would have to allow for a multitude of data types that differ from person to person. This complexity already adds a dimension of subjectiveness to the type of ICT used. The premise for any ICT system in healthcare is the concept of added value [4]. Within the healthcare setting add-value pertains to any ICT system that is placed into a healthcare scenario must allow all participants involved to feel that they are getting greater benefits than previously realised. As the health sector is made up of systems that are continuously changing due to 
various clinical patterns and policies, and information system placed must be able to adapt with the change. This setting is made more complex as stated earlier due to the inherent nature of the developing country setting.

This paper initially explores how access to information is a key part of healthcare and how this is an issue in a developing country setting. The paper then details the developing country setting with an emphasis on how methods that lack any modern technology are being used to implement health awareness programs. The final sections of the paper cover converging technologies and mobile technology and how these two developments can increase the access to healthcare information in a developing country setting.

\section{Access to Information}

In the developing world most people live in rural areas; areas that are sparsely settled away from the influence of large cities [5, 6] However, for many of the people living in these areas the epicentre of healthcare resources and expertise is in the cities. The result is that people need to make their way in urban areas to access their health care, sometimes by the time they get to the urban settlement it is too late. Roughly 75 percent of the World's poorest people live in these rural areas [7]. In these areas people are generally poorer and access to information, services and supplies is limited.

Information has the ability to empower individuals, irrespective of location, and with healthcare empowerment is an integral method to ensure individuals have an equal opportunity to look after their own health [8]. Without access to information, communities in disadvantaged situations are unable to engage in meaningful discussions or make thoughtful decisions regarding healthcare. Information sharing is a model for an individual's interaction with the healthcare system that may significantly improve the overall care of the community [9]. According to McNamara one of the common threads that runs through humans is the "ability to absorb new information, ideas and approaches then putting it into their own context with regards to social, economic and cultural processes. Humans assimilate, adapt and incorporate this new information into their daily realities if it empowers them to better deal with their local situation" [10]. The introduction of ICT's may increase the possibility for these communities to access their information and the use of the information will be adapted to fit in with their setting. The deployment of ICTs in this setting will provide the opportunity for these communities to engage in dialogue and debate around not only local health concerns but also global concerns.

\section{Developing Countries}

According to Whitehead "there is a growing acknowledgement that many countries face serious social inequalities in health" [11]. This divide is more visible in developing countries. In such countries the information poor are those that need the information the most [12]. However before the divide can be fully understood one has to understand the concept of equity in healthcare. Equity is an ethical concept that means different things to different people, it equates to social justice. Equity implies that in the allocation of resources one should look at the need for the resources rather than the privilege of the people/areas receiving these resources [11]. Thus equity in health can be understood as to mean striving to reduce avoidable disparities in physical and psychological wellbeing, as well as in the determinants of that well being. This can be systemically observed between groups of people with different levels of underlying social status. e.g. wealth power and prestige. In virtually every society in the world social privilege varies among groups of people that are categorised not only by economic resources, but also by gender, geographic location, ethnic and religious differences and by age [11]. These inequalities become very pronounced when shifted into the health setting.

In developing countries, inequality is not just based on the grass root level, but by merely being a developing country it is already on the back foot. Lack of infrastructure makes even the most simplest of tasks a monumental effort. However, such countries develop systems to adapt to their situation [13]. The only area where adaptation is still an area of concern is in the area of quality health information. If the information is not portrayed in a correct manner it may lead to negative consequences or lack of uptake. The important point about health information is to make it work as a trigger for behavioural change that will support healthy lifestyles.

Access to quality health information is essential especially in the development of health education, health promotion and in any community is probably regarded as a critical determinant of health. It contributes to enhanced patient care, the development of health policies, management of healthcare systems and the understanding of health conditions by the health consumer. In developing countries the impact of the "know do" gap is prominent [14]. The "know do" gap is the gap that is created from knowledge/awareness to action/behaviour change [15]. The growth of the Internet and especially Web 2.0 technologies, means it has become the universal media for publication, preservation, indexing, dissemination and access to up-todate scientific, technical and factual information. It has become a universal library capable of extending equitable access to quality health information [14].

Thus the Internet is ideally seen as the bridge between inequality of information sharing. However, this is not the case. Despite its growth and the availability of health, scientific information knowledge and evidence there are still numerous barriers that pose a challenge to allow for universal access and thus a promise of an equitable health society. Barriers according to Pandita and Singh include, but not solely made up of [16]:

1. Connectivity - how to expand and maximise the availability and use of different and new media to communicate health information knowledge and evidence to the general public. In particular how to ensure that the information flow and knowledge is ubiquitous to the health consumer.

2. Information technology literacy -how to facilitate the use of technologies to access quality health information through various technological mediums. 
3. Cultural - how to make information that is culturally acceptable and relevant within different settings and domains while keeping the end user in mind.

4. Economic - how to make quality health information open and accessible to all communities.

These are the barriers that affect the health consumer directly. Other barriers are; critical appraisal, copyright, local, national and regional visibility and accessibility and lack of government funding and support [16].

With the existence of these barriers, developing countries have already had to adapt to using various traditional communication methodologies to transfer health information. In developing countries, a large proportion of the population, including health professionals, has no or only poor access to the Internet. Even printed material, such as up-to-date books current journals and newspapers are scarce. In this situation professionals rely on the knowledge they obtained during their training to care for patients, prevent disease and educate patients [17].

With this in mind many developing countries have utilised traditional media for communication health information. The World Health Organisation (WHO) has identified the following traditional media communication sources:

1. Using Soap Operas -Cambodia launched a soap opera with an AIDS theme. This is Cambodia's way of shifting their focus to families as the sex trade is a very prominent industry in this country. They hope to educate families on the dangerous of promiscuous activity as well as the impact of AIDs on peoples' lives while also normalising it to show that a person with AIDs still needs to be taken care of and given attention [18].

2. Using Bicycles - In China most of the television, radio and newspaper is controlled by the government thus it is very hard to promote health information in an acceptable manner. With Beijing having one of the highest bicycle populations in the world, the health boards have used this to their advantage and have used this to pass health information. They do this by dressing up a large group of riders in identical clothing all which has a specific health message and for them to ride together in a single file thus attracting attention [19].

3. Sports Stars against smoking- In Fiji, sports is a common denominator for all people, the passion for sport in Fiji has become a great conduit for health information promotion. The National Centre for Health Promotion has used sports stars in an antismoking campaign. Their campaign slogan "Winners don't smoke. Be smart, don't start" has used the country's netball secondary school teams as well as their relay teams as role models. The impact has been paramount with the campaign posters being visible at schools, community centres and even on phone cards [18].

4. Using puppets- in Laos there are 46 different ethnic groups and five spoken languages with a very low literacy rate thus choosing the correct channel of communications is very difficult. UNICEF have gone back to a traditional medium to promote health education. This done through the use of community theatre. Puppet shows and theatre are strong parts of the Lao culture. Plays and puppet shows have not been customised to carry health messages and have reported a high impact [18].

5. Diverse channels for diverse communities - Malaysia is made up of diverse range of ethnicities. Like most developing countries mainstream media is not always available in rural settings, thus their campaign is focused on using a combination of mediums to pass on the health messages. Mass media adverts have included newspaper print, television, radio, billboards and posters. But these do not always have an effect, as it is very easy to drive past a billboard without reading it. Thus the different strategies include putting slogans on the back seats of buses as they remain at the eye level of the reader for a longer period. Printing health messages on to mugs, placemats, handbooks are some of the other techniques used. Within the rural setting as well, workshops are run at the local communal markets on the importance of hygiene [18].

6. Telephones- In Mongolia, $85 \%$ of health promotion materials are in a printed format but these are not always effective. A very commonly used health promotion tool is the use of hotlines. In fact a major source of information for young people that refuse to discuss safe sex with their parents is done via such hotlines. In 2002 this particular health information hotline received 101080 phone calls [18].

7. Acting - Papua New Guinea is the country that is most affected by the AIDS pandemic in the pacific region with over 45000 people being HIV positive [10]. Mass media has not worked due to the taboo that is associated with talking about sex and sexual transmitted infections. As literacy rate is barely above $50 \%$ print media has no impact on educating a vast majority of the people. The health ministry has gone back to storytelling as a means of promoting HIV/AIDS education. Unlike in Laos, the story telling here is focused totally on getting the message about AIDS it is not a complicated story line and it falls in line with the traditional Wantok system of beliefs in Papua New Guinea $[18,20]$.

8. Using the village elders - This is technique that has been used widely in Africa and also Samoa. It involves educating and informing the village elders and chiefs on the importance of preventing disease through some intervention. Such as condom use in Africa and the importance of good hygiene in Samoa. By getting the chief statesmen to accept and acknowledge these inventions the uptake from the rural surroundings is much higher [21].

9. Pictures - Using pictures as an effective communicating tool has been a major breakthrough in the barriers of understanding. Mapara utilised this effectively in Zambia as a methods of educating communities about HIV/AIDS. The impact was 
immediate as individuals could relate to the items in the pictures. No words were used in the pictures, the pictures themselves told the story [22].

As it can be seen from these few but different examples, the importance of health education and promotion in developing countries has led to the development of innovative methods of communicating health information. Information is power and by informing the lay person about the importance of good health practices it can only stand in good stead for the individual and for community groups. The impact of technology in developing countries is slowly becoming a reality, however the emphasis is still low. As was mentioned at the start, in these countries the information poor will be the last to receive direct interaction with technologies thus the importance is to merge traditional methods with modern techniques and tools.

\section{Converging Communications}

The preceding section detailed the importance of access to information and how this is being overcome utilising traditional media methods in developing countries. The methods used were derived to overcome the lack of access to health information as well as to narrow the gap that has been created by those that have access to technology and those that do not [23]. This section introduces the concept of converging communications and the power that this approach has in narrowing the existing knowledge gap that exists in the developing country setting.

Health professionals, irrespective of the state of the country, depend on their ability to communicate effectively with colleagues and the health consumer in the performance of their healthcare duties. The communication can involve a simple enquiry into how the health consumer is feeling, to the explanation a pharmacist gives to a health consumer on the effects of a certain drug they may be taking [24]. In each case the health professional depends on their ability to communicate effectively in order to get the correct information to the patient. In many cases however the information that is required by the health consumer is needed well before they meet the healthcare professional. It is the old adage of "prevention is better than cure" and in the context of the health sector this prevention message has to be easily understood by the health consumer.

Communication technologies within the health context play an important role. Especially as information flow between healthcare givers and from the health care provider to the health consumer is crucial. With the advent of various technologies though there is a growth in the use of communication technology in consumer empowerment. This empowerment is either self sought or empowerment that is enabled by the health system. This is the empowerment that is missing in a developing country setting, as the power of communication technologies is the speed and updatedness of the information that can be accessed [23].

The previous section showed how convergence of communication methods has been taking place. This has been through traditional technologies and methods, Television, radio, newspaper, acting, chiefs are all communication mediums and methods, and by combining one or more of those methods that is already a convergence of communication methods. However, with the growth of the
Internet and the rapid change of technology this focus now shifts towards the convergence of technological mediums, tools and methods.

The obvious start is to look at the Internet. From starting off as a static, one way dissemination tool, the Internet has transformed dramatically especially with the advent of Web 2.0 which has also resulted in the growth of Health2.0. There is no simple definition for Web 2.0. Broadly put, it is a paradigm shift in the way the Internet is used. Web 2.0 involves a more open approach to the Internet, in particular user-generated content, Blogs, Podcasts, social media, review sites, Wikipedia, etc [25]. Web 2.0 has engineered the use of terminology such as "prosumer" to describe the fact that the traditional Web 1.0 "consumers" of information are now Web 2.0 producers of information [26]. Other common Web 2.0 terms include "architecture of participation" and "people-centric Web" indicating the involvement of users in the production of web content and the collaborative nature of the Web 2.0 environment [25]. Finally, the term "collaborationware" has been applied to the range of Web 2.0 technologies to express their potential for enabling people to work together online [26]. The question though is can this new paradigm shift be incorporated into the health setting and more specifically into a developing country setting? Even though terms like Health 2.0 are flowing out, what exactly does this mean and what is its relevance to the developing country setting?

Health 2.0 has been defined in a number of different ways and there is as yet no authoritative definition of the term. However, the various definitions together with usage on the web itself point to three distinct understandings of the term. With this point in mind, Health 2.0 can be understood as the use of Web 2.0 technologies within healthcare to affect healthcare for the better, particularly in terms of increased consumer participation in health provision [27]. Health 2.0 can also refer to the transformation of the traditional Web 1.0 sites to include Web 2.0 tools and the provision of up-to-date personalised health care information for health consumers [28]. Finally, Health 2.0 can refer to a fundamental change in the way in which healthcare is delivered and conveyed [29]. Two of these points have a direct link on ways to improve or create an exchange of health information within a developing country setting.

Having understood what the terms mean, the next stage is to see what it means for communication technologies. The utopia of a convergent communication tools are Internet enabled phones, for example the Apple iPhone [30] by placing the iPhone into the hands of the health consumer the possibilities seem endless. Being a mobile device, it means that a user is not limited by geographic location, a problem that is faced by rural communities in developing country settings. Thus the user can in any setting, in theory, be able to make contact with a healthcare provider be it via the phone capabilities or through its Wi-Fi capabilities. The iPhone is the perfect platform in which communication technologies are converging onto one medium, however, it is also still a utopian idea for a majority of people to have one as it is an expensive investment, thus is not a real solution for access to health information in a developing country setting. The essence of the iPhone is that it is a mobile device and many mobile devices now, even some of the 
cheaper range models, do have a number different media types associated with them.

The other emerging technology/forum for converging media in a communicative manner is the use of social networks. Networks such as Facebook, MySpace and Bebo are growing in popularity as well as the applications that are being attached to them. Therefore it is quite feasible that the information on the Internet will slowly start being filtered and customised for each user via such social networks. Social networking is becoming a way of healthcare and health education methods for those that have access to the Internet. Why do social networks work? And why will they have an impact in health care? According to Surowiecki "groups are remarkably intelligent and are often smarter than the smartest person in them" [31]. Groups don't have to be led by the smartest person to be smart. When placed in a healthcare scenario, social networks have the following impact. When patients are managing the same condition their own observations are shared, this collective wisdom can yield clinical insights that are more informative than any one patient or healthcare professional will provide [32]. Social networks also have favourable outcomes for healthcare providers in that they can exchange case notes with providers globally and thus get insights that would not be available locally. In a health setting group opinion has a greater impact than individual perspectives. It also allows individuals to start taking ownership of their own health as they are confident that there are social support networks easily available for them to gauge their condition with [32].

With the Internet penetration increasing in most regions the impact of social networks and its form of convergent communications will be a growing trend within healthcare. This is an important paradigm shift that is being observed in the developed world, the same cannot be said for the developing world. Internet usage statistics show that despite the growth of the Internet, there is still a large gap between the developed world and the developing world. The overall percentage of the Internet in Africa is to $6.7 \%$ of the population. This indicates that the Internet is still limited to exclusive groups within the wider population group thus excluding the critical mass again [33]. This links to the thread that this paper is highlighting, despite the increase of use in technology there is a significant lack of access to this technology in the developing country setting.

\section{Mobile Technology}

The convergent theme indicated that mobile technologies are at the fore front of this phenomenon. With the iPhone being the utopian tool, other brands will develop models to rival the iPhone, however till then it is still out of the reach of the average person let alone an individual in a developing country setting. This does not limit the impact that mobile technology can have in healthcare promotion, education and empowerment in disadvantaged settings.

The problem with the Internet, as has been discussed before, is that is very dependent on infrastructure. Developing countries and a number of developed countries still have the dialup copper cables as the mainstay of their Internet. This requires putting up and maintaining the infrastructure [34]. Also the cost of the Internet is not affordable to a large proportion of the rural and under developed populations within developing countries especially [34]. This is where mobile technologies have an advantage The low internet penetration rate suggests that it does not in fact make a feasible solution to bridging the data gap, and whilst mobile phone penetration rates have tripled for developing countries, there is still a gap between access for those in urban and rural areas, however this gap is not as large as the Internet divide $[35,36]$.

Over the last few years mobile telephony has become the principal gateway to ICT access and use, in the developing world, many countries have skipped fixed-line infrastructure and leapfrogged directly into mobile technology. At the beginning of the twenty-first century, the average number of mobile phones per 100 inhabitants in Asia, Africa and Latin America and the Caribbean has risen by $400 \%$ in five years [37]. While developing countries are still lagging behind high-income countries in overall ICT usage and application, the marked increase in mobile phone penetration rates in developing countries especially should be recognised as a potential "digital bridge" for the digital divide. According to the Information economy report 2007-2008 [38] the number of mobile phone subscribers in the developing world has tripled in the last 5 years. The growth has been phenomenal and now these subscribers make up 58\% of all mobile phone users worldwide. Despite the growth being very high, there is still a gap that exists between subscriber numbers and penetration rate. This can be put down to once again the urban and rural setting that plays a big role in different lifestyle approaches, especially in such countries.

However, by comparing Internet penetration figures and the mobile phone penetration figures it can be seen that, especially in the developing world, the penetration rate of mobile phones is significantly higher than the Internet penetration rate [38]. With the increasing mobile coverage and the expansion to even rural areas, something that the Internet has still not done, mobile technology is a more real solution for making health prevention and medical care more effective for the world's poorest people. Rashid and Elder found that Mobiles simply offer the poor with a relatively affordable and accessible option, compared to other ICTs. Also mobile phones help to strengthen social ties among the poor and provide them an opportunity to communicate in the case of emergencies [39].

Mobile phones increase the ability to contact individuals who are busy, remote or always on the move. In healthcare, mobile phones offer opportunities for efficient and effective service delivery. The benefits that are offered by mobile technologies are not limited to rural areas only. Privacy and confidentiality, especially in healthcare, is important. Mobile phones are more often than not personal device and not shared devices, thus it is easier for people to access and address personal information on their devices. Their use by teenagers to obtain healthcare information via phone calls, has been documented to be a success as they are more aware of the fact that no one can over ear their conversation as they can so go into secluded areas to talk.

One of the key functions of mobile phones that make it suitable for healthcare delivery is the use of its Short Message Service (SMS) function. The SMS itself is a simple form of data transfer usually communicated person to person but which can also be sent computer to person and vice 
versa. The internet and email have some of the same characteristics but, in the context of health care management, the fact that the device is held by, and is personal to, the user (patient) is key. Mobility has the further potential to increase efficiency by the ability to reach the patient directly. Apart from it's widespread usage, the SMS application also has many further characteristics that make it appropriate for use in a healthcare context [39].

With this in mind, the use of mobile phones can be regarded as a very real medium of passing health information to the health consumer. From simple things like appointment reminders to more complex things like personalised health information about the user. The high penetration rate of mobiles in most social groups makes it feasible for SMS to be used as a core communications channel. In 2005 there were a total of 1.25 trillion SMS messages sent globally [39]. Taken into account that this was in 2005 and the growth from then has increased it can be fair to say that globally SMS messaging can be used as a very effective health care communication tool, even in developing countries.

The 'one-to-many' feature of SMS systems means that messages can be sent to many recipients simultaneously and potentially in several different languages[39]. These messages can also be pre-written, minimising time and effort in data entry and communication for providers, but it also allows manual or custom messaging. Messages are also held until the recipient can read them thereby increasing convenience. Furthermore, messages sent to a mobile phone that is switched off are stored at an SMS Centre and delivered when the handset is switched on again.

With the development of not just the Internet enabled phones, but also the growth of websites dedicated to offering mobile services, the power of the SMS is increasing. For example, Twitter is a free micro-blogging service that allows its users to send updates which are text-based posts of up to 140 characters in length. Updates are displayed on the user's profile page and delivered to other users who have signed up to receive them. The sender can restrict delivery to those in his or her circle of friends (delivery to everyone being the default). Users can receive updates via the Twitter website, instant messaging, SMS, RSS, email or through other social networking websites [29]. The impact of this easy to use interface within the health sector may have many positive impacts. One such impact would be the ease of informing a multiple number of users on viral outbreaks such as SARS or Bird Flu, in a simple and effective manner. Minimum effort with maximum results is something that mobile technologies can make a reality.

\section{CONCLUSION}

ICT is here to stay, however unless it is harnessed in a manner that can benefit populations irrespective of their socio-economic standing, gender and age it will carry the failings of traditional methods that have been incorporated into healthcare awareness in the past. The vital importance of access to health information by all is justified through concepts of equality and consumer empowerment. By utilising its convergence abilities, the use of ICT through mobile phones and social networks can be used to reach previously unreachable populations with customisable information. Learning's from using traditional methods can be taken onboard and used to develop healthcare and awareness plans using ICT as a medium and not as the sole solution.

\section{REFERENCES}

[1] Ministry of Economic Development, Digital Strategy2.0. 2007 Available from: http://www.digitalstrategy.govt.nz/upload/Docum ents/Digital $\% 20$ Strategy $\% 202.0 \% 20$ FINAL.pdf [Accessed on October 10 2009].

[2] Walshman G, Sahay S. Research on Information Systems in Developing Countries: current landscape and future prospects. Inform Technol Dev 2006; 12: 7-27.

[3] Chetley A, Ed. Improving health, connecting people: the role of ICTs in the health sector of developing countries. 2006. Available from http://www.healthlink.org.uk/PDFs/infodev_frame.pdf [Accessed on July 12, 2009].

[4] Wood D. The added-value concept. 1978. Available from http://www.emeraldinsight.com/Insight/viewPDF.jsp?contentType $=$ Article $\&$ Filename $=$ html/Output $/$ Published/EmeraldFullTextArticl e/Pdf/0040200501.pdf [Accessed on October 12 2009].

[5] Lund VK. The healthy communities movement: bridging the gap between urban planning and public health. Available from: http://www.asu.edu/caed/proceedings99/LUND/LUND.HTM [Accessed October 11, 2009].

[6] Fotso JC. Child health inequities in developing countries: differences across urban and rural areas. Int J Equity Health 2006; 5: 9 .

[7] Ki-moon B, Zapatero R. Food security: Our forgotten crisis. International Herald Tribune. 2009. Available from http://www.un. org/sg/articleFull.asp?TID $=90 \&$ Type $=$ Op-Ed [Accessed on July 12, 2009].

[8] Custodio R, Gard AM, Graham G. Addressing health disparity by improving quality, increasing access, and developing workforce. $\mathrm{J}$ Health Care Poor Undeserved 2009; 20: 301-7.

[9] D'Allesandro D, Dosa N. Empowering children and families with information technology. Arch Pediatr Adolesc Med 2001; 155: 1131-6.

[10] McNamara K, Ed. Improving health, connecting people: The role of ICTs in the health sector of developing countries. 2006. Available from http:/unpan1.un.org/intradoc/groups/public/docum ents/un-dpadm/unpan036467.pdf [Accessed April 7 2009].

[11] Whitehead M. A typology of actions to tackle social inequalities in health. J Epidemiol Commun Health 2007; 61: 473-8.

[12] Prata N. Making family planning accessible in resource-poor settings. Philos Trans R Soc Lond B Biol Sci 2009; 364(1532): 3093-9.

[13] Morel C, Broun D, Dangi A, et al. Health innovation in developing countries to address diseases of the poor. Innov Strategy Today 2005; $1: 1-15$.

[14] Braveman P, Tarimo E. Social inequalities in health within countries: not only an issue for affluent nations. Soc Sci Med 2002; 54(11): 1621-35.

[15] World Health Organisation. The Know do gap. Proceedings from Gap Knowledge translation in global health. 2005 December 8; Geneva, Switzerland -. Available from http://www.who.int $/ \mathrm{kms} / \mathrm{ev}$ ents/ShademaniR_KT-NIPH_PH\%20Consultation_8Dec05.pdf [Accessed on July 1 2009].

[16] Pandita N, Singh S. Barriers to equitable access to quality health information with emphasis on developing countries. Proceedings of the Making the eHealth Connection: Global Partnerships, Local Solutions conference; 2008 July 13-8 August; Bellagio, Italy; 2008.

[17] Mouhouelo P, Okessi A, Kabore MP. Where there is no Internet: delivering health information via the Blue Trunk Libraries. PLoS Med 2006; 3: e77.

[18] World Health Organisation. Choosing the Channels of Communication. Available from http://www.wpro.who.int/internet/ resources.ashx/TFI/choosing+the+channels + of + communication.pdf [Accessed on July 1, 2009].

[19] Blair M, Armstrong R, Murphy M. The 360 degree brand in Asia: creating more effective marketing communications, John Wiley and Sons, Asia, 2003

[20] UNAIDS. Papua New Guinea: country progress report. Available from: http://data.unaids.org/pub/Report/2008/papua_new_guinea 2008_country_progress_report_en.pdf [Accessed on July 12, 2009] 
[21] Allen T, Heald S. HIV/AIDS policy in Africa: What has worked in Uganda and what has failed in Botswana? J Int Dev 2004; 16: 1141-54.

[22] Mapara EM. Pictures as a Health Promotion Strategy in addressing HIV/AIDS in the Developed Countries. 2006 Available from http://www.ahpn.org/downloads/publications/Pictures_as_a_Health _Promotion_Strategy_Adobe.pdf [Accessed on July 12, 2009]

[23] Schilderman T. Strengthening the knowledge and information systems of the urban poor. 2002 DFID/ITDG. Available from www.id21.org/society/s4bts1g1.html. [Accessed October 13 2009].

[24] Stewart M. Effective physician-patient communication and health outcomes: a review. Can Med Assoc J 1995; 152: 1423-33.

[25] O'reilly T. What Is Web 2.0? Design patterns and business models for the next generation of software. 2005. Available from http://oreilly.com/web2/archive/what-is-web-20.html [Accessed on July 1, 2009].

[26] Doherthy, I. 'Web 2.0: a movement within the health community', health care and informatics review online, 2008.. Available from http://www.hinz.org.nz/journal/2008/06/-Web-2-0---A-MovementWithin-The-Health-Community/991 [Accessed on July 1, 2009].

[27] Eysenbach G. Medicine 2.0. Social Networking, Collaboration, Participation, Apomediation, and Openness. J Med Internet Res 2008; 10: e22.

[28] Bos L, Marsh A, Carroll D, Gupta S, Rees M. Patient 2.0 Empowerment. In Arabnia HR, Marsh A, Eds. Proceedings of the 2008 International Conference on Semantic Web and Web Services Las Vegas, Nevada, United States of America. July 14-17, 2008.

[29] Hawn C. Take two aspirin and tweet me in the morning: how twitter, facebook, and other social media are reshaping? Health Aff 2009; $2: 361-9$.
[30] Grossman L. Invention of the year: the iPhone. Oct 302007. Available from http://www.time.com/time/specials/2007/article/ 0,28804,1677329_1678542_1677891,00.html [Accessed on January 1,2009$]$.

[31] Surowiecki J. The wisdom of crowds: why the many are smarter than the few and how collective wisdom shapes business, economies, societies and nations, Random House Inc. 2005.

[32] Sarasohn-Kahn J. The wisdom of patients: health care meets online social media. Available from http://www.chcf.org/documents/ chronicdisease/HealthCareSocialMedia.pdf Oakland, CA: California Healthcare Foundation; 2008.

[33] Internet Usage Statistics. Available from http://www.internetworld stats.com/stats.htm [Accessed 29 September 2009].

[34] Edejer TT-T. Disseminating health information in developing countries: The role of the Internet. Br Med J 2000; 321: 797-800.

[35] Fong M. Digital divide between urban and rural regions in China Elec J Info Sys Dev Count 2009: 36(6): 1-12.

[36] James J. From the relative to the absolute digital divide in developing countries. Technol Forecast Soc Change 2009; 76(8): 1124-9.

[37] UNCTAD. Information Economy Report 2007-2008 Science and technology for development: the new paradigm of ICT. Geneva: United Nations Publication 2007.

[38] McQueen D, Newman M, Patterson G, Roberts M, Nick L. Mobile industry Outlook 2007; Informa Media And Telecom.

[39] Atun RA, Sittampalam SR. A review of the characteristics and benefits of SMS in delivering healthcare. the role of mobile phones in increasing accessibility and efficiency in healthcare., Vodafone Policy Paper Series 2006; No. 4: 18-28. 